\title{
Enhanced Human Memory Consolidation With Post-Learning Stress: Interaction With the Degree of Arousal at Encoding
}

\author{
Larry Cahill, ${ }^{1}$ Lukasz Gorski, and Kathryn Le \\ Department of Neurobiology and Behavior, and Center for the Neurobiology of Learning and Memory, University of California, \\ Irvine, California 92697-3800, USA
}

\begin{abstract}
Abundant evidence indicates that endogenous stress hormones such as epinephrine and corticosterone modulate memory consolidation in animals. We recently provided the first demonstration that an endogenous stress hormone (epinephrine) can enhance human memory consolidation. However, these findings also suggested that post-learning stress hormone activation does not uniformly enhance memory for all recently acquired information; rather, that it interacts with the degree of arousal at initial encoding of material in modulating memory for the material. Here we tested this hypothesis by administering cold pressor stress (CPS) or a control procedure to subjects after they viewed slides of varying emotional content, and assessing memory for the slides 1 wk later. CPS, which significantly elevated salivary cortisol levels, enhanced memory for emotionally arousing slides compared with the controls, but did not affect memory for relatively neutral slides. These findings further support the view that post-learning stress hormone-related activity interacts with arousal at initial encoding to modulate memory consolidation.
\end{abstract}

Abundant evidence dating to the initial findings of McGaugh in the 1950's documents that treatments administered after learning, such as drug injections or brain stimulation, can modulate (enhance or impair) memory consolidation processes (McGaugh 2000). Abundant evidence also documents that endogenous stress hormones such as epinephrine and corticosterone also modulate consolidation in animals (McGaugh 2000; Roozendaal 2000). And recently, we provided the first demonstration that an endogenous stress hormone can modulate human memory consolidation (Cahill and Alkire 2003). This evidence forms a cornerstone of the view that enhanced memory for emotionally stressful events results from the enhancing effects of stress hormones on consolidation processes (Gold and McGaugh 1975).

A widespread assumption has been that stress hormone action rather uniformly modulates memory consolidation for recently acquired information. Livingston (1967), originator of the "Now print!" metaphor about arousal's influence on memory storage, made this assumption explicit by suggesting that after an arousing/significant event "the brain 'prints' remembrance of all events immediately preceding" and that "following a ... "Now print!' order, everything that has been ongoing in the recent past will receive a 'Now print!' contribution in the form of a growth stimulus or a neurohormonal influence that will favor future repetitions of the same neural activities" (italics added). In contrast to this view, our recent findings with post-learning epinephrine infusions suggested that the degree of arousal associated with initial encoding of material helped determine whether postlearning epinephrine effectively modulated consolidation of the material (Cahill and Alkire 2003). Our results resembled those of Buchanan and Lovallo (2001), who found that pre-learning administration of cortisol enhanced long-term memory for relatively arousing pictures, but not for relatively neutral pictures.

\footnotetext{
'Corresponding author.

E-MAIL Ifcahill@uci.edu; FAX (949) 824-5244.

Article and publication are at http://www.learnmem.org/cgi/doi/10.1101/ Im.62403.
}

The goal of the present experiment was to further examine the hypothesized interaction between post-learning stress and arousal at initial encoding using cold pressor stress (CPS). Although it has not previously been used to modulate memory consolidation processes, CPS is a widely used technique in medical research (Lovallo 1975) and induces a robust, reliable stress hormone response, including cortisol (CORT) activation. We administered CPS or a control procedure to subjects after they viewed a series of slides very similar to those used in our previous study of epinephrine's influence on consolidation (Cahill and Alkire 2003). If post-learning stress, and concomitant stress hormone activation, interact with arousal associated with initial encoding of slides to modulate memory consolidation of the slides, then CPS administered immediately after learning should enhance long-term memory for relatively arousing slides, but not affect memory for relatively neutral slides in the same subjects.

\section{RESULTS}

A total of 11 subjects out of 59 total were excluded from analysis for various reasons: six because of indications that they were aware that they would experience a memory test, two for technical problems during the experiment, one because her baseline CORT levels were over four standard deviations (SD) above the group mean, and two because they appeared by their ratings to have experienced virtually no arousal response to the CPS procedure, rating the experience more than 2 SD below the group average. Thus the final number of subjects was 25 in the CPS group (15 female, 10 male) and 23 in the control group (19 female, 4 male).

\section{Cortisol and Emotional Response to CPS}

As expected, the ratings of discomfort revealed that the CPS procedure produced a far greater level of discomfort than did the control procedure $(t[46]=23.3, P<.0001)$. Figure 1 shows the results from the salivary CORT analysis. As expected, there was no difference between the CPS and control groups in CORT level at baseline. After the arm immersion procedure, CORT levels were significantly higher in the CPS group than in the control 


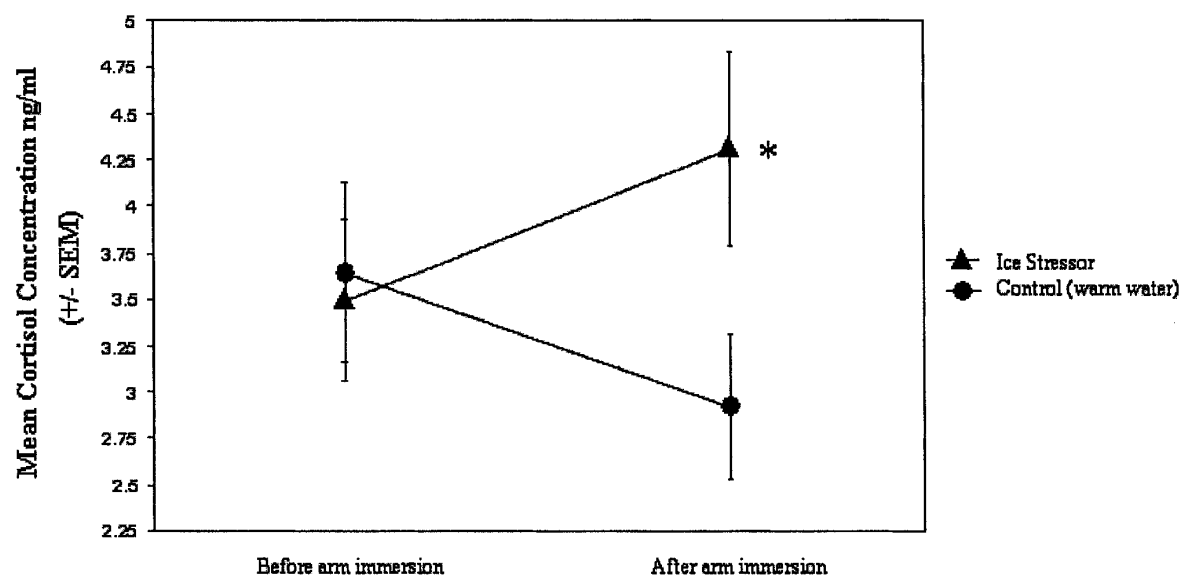

Figure 1 Average ( \pm SEM) salivary cortisol response to CPS or control arm immersion. * $=P<.05$ from the corresponding control value.

group $(\mathrm{t}[46]=1.39, P<.05)$. In addition, CORT levels rose significantly after arm immersion in the CPS group compared with baseline $(\mathrm{t}[24]=-3.56, P<.002$, paired $\mathrm{t}$-test), but dropped significantly in the control group $(\mathrm{t}[22]=3.31, P<.005$, paired t-test). The average amount of time subjects kept their arm in the ice water was $95.4 \pm 67.1 \mathrm{sec}$.

\section{Recall of All Slides}

CPS produced a nearly significant increase $(\mathrm{t}[48]=1.96, P=.056)$ in total recall of the 21 slides compared with the control procedure, with CPS subjects recalling a mean \pm SEM of $9.2 \pm 0.40$ slides compared to $7.9 \pm 0.50$ slides recalled for the control group. The CPS group also recalled on average more details from the slides as a whole $29.8 \pm 2.3$ than did the control group $24.5 \pm 2.2$, although this effect was not statistically reliable $(\mathrm{t}[46]=1.65, P=.10)$.

\section{Recall of Arousing Versus Neutral Slides}

The data revealed very different effects of the CPS and control procedures on recall of arousing versus neutral slides, as defined by the self-reported emotional reactions to the slides. As shown in Figure 2, CPS subjects recalled significantly more of the arousing slides than did the control subjects $(\mathrm{t}[46]=2.59, P<.02)$. In contrast, CPS subjects did not differ from controls in recall of neutral slides $(\mathrm{t}[46]=-0.49, P \gg .10)$. When the analysis was restricted to only the female subjects, the same conclusions were obtained: Women in the CPS group recalled significantly more arousing slides than did the controls $(\mathrm{t}[32]=2.14$, $P<.05)$ but did not differ from them in recall of neutral slides $(\mathrm{t}[32]=0.49$, $P \gg .10)$.

CPS also affected the number of details recalled from each slide. As shown in Figure 3, CPS subjects recalled a significantly greater number of details from the arousing slides than did control subjects $(\mathrm{t}[46]=2.73, P<.01)$, but the CPS and control subjects did not differ in recall of details from neutral slides $(\mathrm{t}[46]=-.23, \mathrm{n} . \mathrm{s}$.$) . Interestingly, in sub-$ jects receiving the control arm immersion, significantly fewer details were recalled from the arousing compared with the neutral slides $(\mathrm{t}[22]=-2.97, P<.01$, paired t-test). The number of details re- called from arousing versus neutral slides did not differ significantly in the CPS group $(\mathrm{t}[24]=1.31, P>.10)$.

\section{Relationship Between Cortisol Response and Memory}

The relationship between the degree of cortisol response and memory was assessed in the CPS subjects by correlating the change in cortisol levels (determined by subtracting the level at baseline from that after immersion) with either (1) the total number of slides recalled or (2) the percent or arousing slides recalled, using a Pearson product correlation. Neither memory score correlated with the cortisol change $(\mathrm{r}=-0.19$ for total recall, $\mathrm{r}=0.06$ for arousing recall, $P \gg .10$ in both cases). There was also no significant correlation between ratings of discomfort induced by the CPS and total slide recall, neutral slide recall, and arousing slide recall ( $P \gg .10$ in all cases). Ratings of discomfort in the CPS group did correlate significantly with the cortisol response $(r=0.58, P<.002)$, although the length of time subjects held their arm in the ice water did not $(r=0.23$, n.s.).

\section{DISCUSSION}

This experiment tested the hypothesis that post-learning stress hormone activation does not uniformly modulate memory consolidation for all recently acquired information; rather, that it interacts with the degree of arousal associated with initial encoding of stimuli to modulate consolidation of those stimuli. We tested this hypothesis by examining the effects of a well known stressor-cold pressor stress-administered after subjects viewed a series of slides of varying arousal content on long-term memory for the slides. As expected, CPS significantly activated an endogenous stress hormone response, as indicated by salivary CORT levels. And as predicted by the hypothesis, CPS significantly enhanced memory for slides defined by the subjects as relatively emotionally arousing, but did not effect memory for slides defined as relatively emotionally neutral. These findings therefore converge with those of our previous study of epinephrine's effect on human memory consolidation (Cahill and Alkire 2003) in suggesting that post-learning stress hormone activation interacts

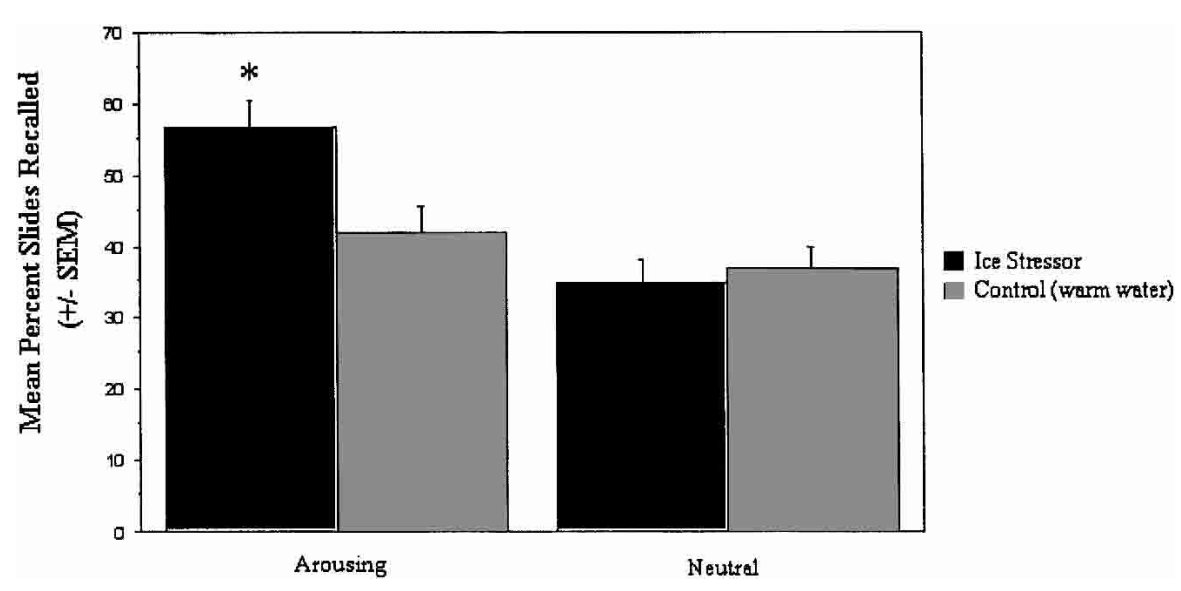

Figure 2 Average ( \pm SEM) percent recall of slides defined as arousing and as neutral by the CPS and control groups. ${ }^{*}=P<.02$ from corresponding control value. 


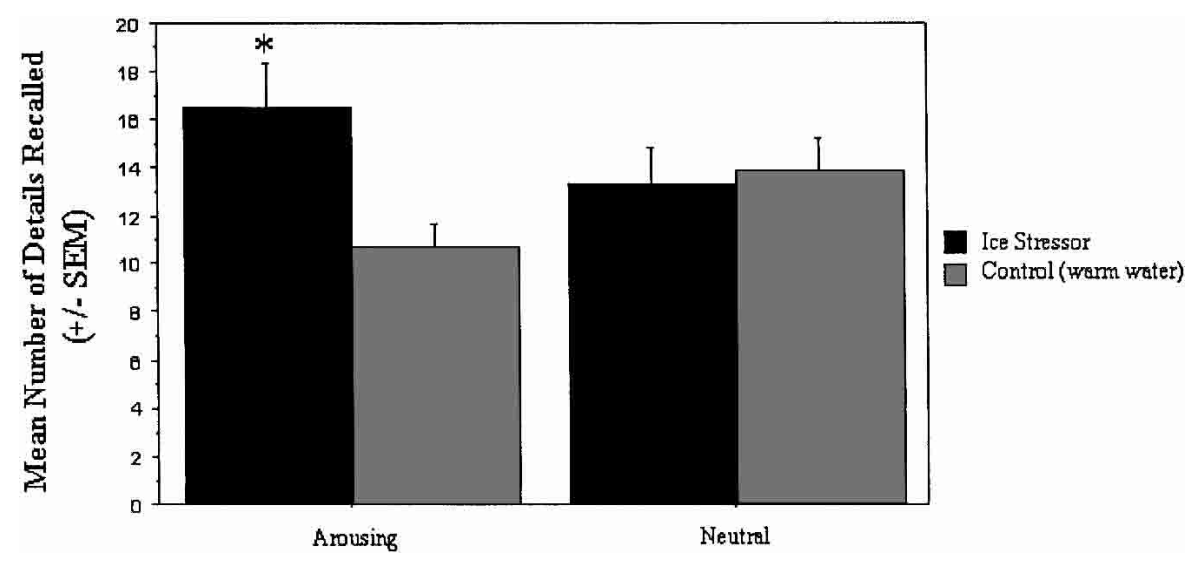

Figure 3 Average ( \pm SEM) number of details recalled from both arousing and neutral slides by the CPS and control groups. * $<.01$ from corresponding control value.

with the degree of arousal at initial encoding of experiences to modulate memory consolidation for the experiences.

There are other suggestions that drug administration in humans selectively enhances memory for relatively arousing material, although the issue has not, to our knowledge, been systematically explored. For example, selective actions of both glucose (Messier et al. 1998) and the cholinergic antagonist scopolamine (Crow 1979) on memory for the presumably relatively arousing (Cahill and Alkire 2003) primacy portion of word lists have been reported. Similarly, Buchanan and Lovallo (2001) reported that pre-learning cortisol administration selectively enhanced longterm memory of arousing pictures. Conversely, de Quervain et al. (2000) found that post-training corticosterone administration in humans did not effect memory for non-arousing material. Finally, but somewhat less directly related, Nielson et al. (1996) reported that post-learning muscle tension arousal enhanced memory for words only when 'priming' tension was administered before learning. Collectively, each of the above findings is consistent with the present study in suggesting that post-learning hormone/stress effects on memory depend in some manner on the degree of arousal associated with the initial learning. Thus these data challenge the widespread assumption, made explicit by Livingston (1967), that post-learning stress and stress hormone action will modulate memory for all recently acquired information.

It should be emphasized that because CPS was administered after learning, its enhancing effect on memory cannot be attributed to actions on attentional, emotional, perceptual, or encoding processes during slide presentation, and must have resulted from some action on memory storage processes. Because CPS also significantly, as expected, activated the endogenous stress response (assessed via salivary CORT levels), the findings provide new evidence consistent with the proposal (Gold and McGaugh 1975) that endogenous hormones released by emotionally stressful events modulate memory consolidation of the events, and support the view that endogenous stress hormones are an essential component of an endogenous memory modulating system for emotionally arousing events that helps insure memory strength that is, in general, proportional to memory importance (Cahill and McGaugh 1998). More generally, these findings add to the studies demonstrating modulation of human memory consolidation with post-training manipulations (e.g., Weingartner and Parker 1984; Colrain et al. 1992; Manning et al. 1992; Soetens et al. 1995; Scholey et al. 1998; Southwick et al. 2002; Cahill and Alkire 2003).

We did not detect a correlation in this experiment between the change in CORT level induced by CPS and memory either for the total number of slides recalled, or for the percentage of arousing slides recalled, as may be expected if CORT activity is causally related to enhanced memory consolidation, as suggested by extensive animal research (Roozendaal 2000). The failure to establish a correlation in this instance may have resulted from the time at which we assessed CORT in saliva post-CPS. Although the timepoint used (10 min after CPS) produced evidence of a significant increase in cortisol levels with CPS, it may not have detected the peak cortisol change, which could have occurred 20-30 min after CPS (Roozendaal 2000). Future studies examining the cortisol response to CPS at different time points after the stressor may detect a significant relationship between cortisol activation and memory.

CPS has been very widely used in a variety of medical research domains (most notably cardiovascular research) for over 50 years (Lovallo 1975). Thus it is noteworthy that these findings constitute the first demonstration that CPS can enhance human memory consolidation. The findings therefore indicate that CPS can be an effective experimental tool for those interested in neural mechanisms of memory consolidation in humans, and its modulation by stressful experiences.

Recent findings point to substantial sex-related influences on neural mechanisms of memory for emotional events (Shors 1998; Cahill et al. 2001; Canli et al. 2002; Cahill and van Stegeren 2003). As noted in the results, a highly similar effect of CPS on memory for arousing slides was found when the analysis was restricted to female subjects. However, the present study involved too few male subjects to allow conclusions about whether the same effect existed in the male subjects alone. Thus the question of whether gender may influence the effects of post-learning CPS and concomitant stress hormone release on memory consolidation in humans remains open, and in our view merits attention in future studies. Evidence suggests that the sex of the subjects may influence the correlation between cortisol activation in response to CPS and memory (Wolf et al. 2001), as well as the effect of CPS on recall of details (Cahill and van Stegeren 2003). At minimum, the available evidence suggests to us that identical effects of CPS on memory consolidation in men and women should not be assumed.

In this experiment, significantly fewer details were recalled in control subjects for arousing compared with neutral slides (Fig. 3). The selective enhancing effect of CPS on memory for details from arousing slides needs to be considered relative to this finding. The fact that fewer details were recalled from the arousing compared with neutral slides in the control subjects may be related to attentional narrowing hypothesized to occur with heightened arousal (the well known "Easterbrook" effect). Thus it is possible that post-learning CPS enhanced detail memory at least in part by blocking an impairing effect on consolidation produced by an Easterbrook effect. Furthermore, it is conceivable that subject sex further influences this effect. Possibilities such as these seem to us to merit attention in future work.

In summary, the present findings demonstrate that CPS administered immediately after viewing a series of slides enhanced long-term memory consolidation for relatively emotionally arousing slides, but not for relatively neutral slides, compared with the effects of the control procedure. CPS also significantly 
enhanced salivary CORT levels, consistent with the possibility that CORT activity influenced memory consolidation. The findings support the hypothesis derived from a previous study of epinephrine's effect on human memory consolidation (Cahill and Alkire 2003) in suggesting that post-learning endogenous stress hormone activity interacts with the degree of arousal at initial encoding of material to modulate consolidation of that material into long-term memory.

\section{MATERIALS AND METHODS}

\section{Subjects}

A total of 59 healthy subjects, all UCI students, average age ( \pm SEM) of $20.1 \pm 3.6$ years, 51 right-handed and eight lefthanded, participated in this study. All provided written informed consent prior to participating according to the dictates of the Institutional Review Board of UC Irvine and received course credit for their participation. All subjects were individually tested.

\section{Materials}

The 21 slides used in this study were taken from the International Affective Picture System (IAPS; Lang et al. 1999), a collection of images widely used in psychophysiological research. Slides were chosen for their low-to-moderate arousal quality as determined by the independent IAPS ratings. The average standardized rating (see Lang et al. 1999) of arousal for the slides was $4.64 \pm 0.31$, and the average valence rating was $5.15 \pm 0.45$, and involved only slides with negative valence. The slides were shown in random order to each subject with the exception of the first slide, for which all subjects saw one of three neutral slides (a light bulb, a fork, or a truck), randomly assigned across subjects. An initial neutral slide was used to avoid potential ceiling effects in recall that might result from the combined effects of a primacy and arousal on memory. Each slide was presented for $15 \mathrm{sec}$, with no blank screen in-between slides. Slides were presented on a standard computer screen located approximately 3.5 feet in front of the subject. The following IAPS slides were used: 717, 253, 303 , $623,237,708,156,503,130,589,726,109,957,907,461,713$, $226,265,900,167,322$. The average emotional reaction rating of the CPS and control subjects for the slides did not differ $(\mathrm{t}[46]=1.4, P>.10)$.

\section{Procedure}

To reduce the impact of diurnal variation in cortisol levels, all testing was performed between noon and 5:00 pm, and the experiment was run across the CPS/control groups. Before the experiment began, each subject was asked to wash their hands and rinse their mouths with water in preparation for providing a saliva sample. The subject then sat comfortably in a reclining chair and was connected via right-hand index and middle fingers to a pulse plethysmograph (MP100, BioPac Instruments). This allowed for potential measurement of heart rate changes in response to slide viewing, and provided a necessary ruse to convince the subjects that the study was about emotional reactions to stimuli, thereby reducing the likelihood that they would suspect a subsequent memory test. Subjects were told that they would likely find some of the slides pleasant, some unpleasant, and some neutral. They were further told that, when cued by the experimenter ( $5 \mathrm{sec}$ after each slide onset) they were to name the slide using a word or short phrase, and that the content of each slide would be easily identifiable (e.g., a tennis player). Subjects were asked to identify each slide to insure that each slide was encoded by each subject. At the appropriate moment, the room lights were dimmed, and slide presentation began. Subjects' naming responses for each slide were recorded.

Immediately after slide presentation, the subject immersed his or her left arm up to and including the elbow in either warm $\left(37^{\circ}-40^{\circ} \mathrm{C}\right)$ or ice-cold $\left(0^{\circ}-3^{\circ} \mathrm{C}\right)$ water. Although all subjects were informed at the outset that they may be asked to place their arm in ice water, no subject knew in which group they were assigned until immediately before arm immersion. Those in the warm condition kept their arm in the water until instructed by the experimenter to remove their arm, which occurred pseudorandomly across subjects 1,2 , or 3 min after arm immersion. Those in the ice-water condition were told that, because the procedure can be extremely uncomfortable, they should keep their arm in the water for as long as possible, not exceeding $3 \mathrm{~min}$, and that they could remove their arm at their discretion. Those who kept their arm in for $3 \mathrm{~min}$ were instructed at that point to remove their arm from the water. After arm immersion, each subject rested for 3 min with their arm covered by a blanket. After the rest period, the room lights were again turned on, and the subject asked to rate the level of discomfort they experienced during water immersion. To do this, they were first asked to recall the most intense physical pain they had ever experienced, and rate this experience by appropriately marking a 0 to 10 scale, with 0 denoted as "no pain or discomfort" and 10 denoted as "the worst pain or discomfort imaginable." This was done to 'calibrate' each subject to the scale. They next rated the peak pain/discomfort they experienced during arm immersion on the same scale.

\section{Saliva Collection}

Saliva samples were collected from each subject for analysis of cortisol levels immediately before slide viewing began (baseline) and $10 \mathrm{~min}$ after removal of their arm from water. Saliva was collected using Salivette tubes (Sarstedt). For each sample, the subject first placed the cotton swab provided in each Salivette tube under his/her tongue, or between a cheek and teeth, for 1 min to produce saliva. The swab was then placed back in the salivette tube, and the samples centrifuged for $20 \mathrm{~min}$ and stored at $-40^{\circ} \mathrm{C}$ until assayed.

\section{Memory Testing}

One wk after slide viewing, each subject returned to the laboratory expecting to view a new set of slides. Instead, they received a free-recall test of their memory for the slides viewed $1 \mathrm{wk}$ earlier. Subjects were told that they had as much time as they needed to recall, and write down, as many of the slides from the previous week as they could. All subjects finished within $15 \mathrm{~min}$. After they finished, subjects were next asked to list, for each slide recalled, as many details as they could recall. They were instructed to write one detail per line of the scoring sheet (thus allowing the definition of a detail to be made by the subjects in all cases), and that no detail was too small to list. The subjects again understood this task had no time limit. After they had written all they felt they could remember, the experimenter asked them if they were sure they were finished, and if there was anything else they felt they might recall. This added encouragement procedure was used because in our experience it often elicits additional accurate recall from subjects. After the memory assessment, subjects again viewed each picture seen 1 wk earlier and rated how arousing they found the slide to be on a 1 to 9 scale, with 1 as "calming/relaxing," 5 as "neutral," and 9 as "exciting/agitating." Subjects were then asked whether they knew or suspected that their memory would be tested, and whether they had seen any of the slides before (all indicated that they had not seen any of the slides previously). After the experiment, subjects were fully debriefed as to the nature of the study.

\section{Cortisol Analysis}

Salivary cortisol concentrations were measured using a Cortisol ELISA (enzyme-linked immunosorbent assay) Kit (DRG International), in which $100 \mu \mathrm{L}$ of saliva was added to the appropriate wells in the anti-cortisol rabbit antibody-precoated microplate. To each well, $200 \mu \mathrm{L}$ of cortisol horseradish peroxidase conjugate was added, except in the substrate blanks. The plate was then incubated for $1 \mathrm{~h}$ at room temperature, and washed three times with wash solution. Two hundred $\mu \mathrm{L}$ of tetramethylbenzidine (TMB) was added to the wells, and the plates were then incubated at room temperature for 30 min to allow for color development. The reaction was then stopped by the addition of $100 \mu \mathrm{L} 0.5 \mathrm{M}$ sulfuric acid, and the plates were read at $450 \mathrm{~nm}$ with a Multiskan Ascent microplate photometer (Thermo Labsystems Oy). Cortisol 
concentrations in each sample were determined from a calibration curve.

\section{Memory Analysis}

Total slide recall was determined by counting the number of slides recalled by each subject. Subjects were credited with accurate recall of a slide when their description of it could unequivocally be assigned to a specific slide that they had seen. "Arousing" and "neutral" slides were determined by a median split of the ratings given by each subject to the slides. Slides receiving a rating by a subject at or below the median arousal rating for that subject were defined as "neutral," whereas slides rated above the median were defined as "arousing." The percent of arousing and neutral slides recalled by each subject was then determined, and these percent values were averaged across subjects to create a mean percent recall score for both arousing and neutral slides in both the CPS and control conditions. These values were compared with Student's t-tests (unpaired, two-tailed). The number of correct details recalled per slide was also determined for each subject. In all cases, a subject's responses were readily identified (by L.G.) as accurate or, in a small percentage of cases, inaccurate details of the corresponding slide. The total number of details recalled by each subject was determined, both for the arousing and for neutral slides. An average score across subjects in each group was then computed using this total detail score.

Analysis of heart rate changes in a large subset of subjects in this experiment detected virtually no heart rate change in response to either the neutral or arousing slides in subjects in these conditions, perhaps due to the relative insensitivity of the pulse plethysmograph technique. Therefore, these data were not useful as a potential additional measure of arousal in response to the slides, and thus we did not relate them to memory performance.

\section{ACKNOWLEDGMENTS}

Supported by NIMH grant MH-57508 to L.C.

The publication costs of this article were defrayed in part by payment of page charges. This article must therefore be hereby marked "advertisement" in accordance with 18 USC section 1734 solely to indicate this fact.

\section{REFERENCES}

Buchanan, T.W. and Lovallo, W.R. 2001. Enhanced memory for emotional material following stress-level cortisol treatment in humans. Psychoneuroendocrinol. 26: 307-317.

Cahill, L. and Alkire, M. 2003. Epinephrine enhancement of human memory consolidation: Interaction with arousal at encoding. Neurobiol. Learn. Mem. 79: 194-198.

Cahill, L. and McGaugh, J.L. 1998. Mechanisms of emotional arousal and lasting declarative memory. Trends Neurosci. 21: 294-299.

Cahill, L., Haier, R.J., White, N.S., Fallon, J., Kilpatrick, L., Lawrence, C., Potkin, S.G., Alkire, M.T. 2001. Sex-related difference in amygdala activity during emotionally influenced memory storage. Neurobiol. Learn. Mem. 75: 1-9.

Cahill, L. and van Stegeren, A. 2003. Sex-related impairment of memory for emotional events with $\beta$-adrenergic blockade. Neurobiol. Learn.
Mem. 79: 81-88.

Canli, T., Desmond, J., Zhao, Z., and Gabrieli, J. 2002. Sex differences in the neural basis of emotional memories. Proc. Natl. Acad. Sci. 99: 10789-10794.

Colrain, I.M., Mangan, G.L., Pellett, O.L., and Bates, T.C. 1992. Effects of postlearning smoking on memory consolidation. Psychopharmacology (Berl) 108: 448-451.

Crow, T. 1979. In Brain mechanisms in memory and learning: From the single neuron to man (ed. M.A.B. Brazier), pp. 269-275. Raven Press, NY.

de Quervain, D.J., Roozendaal, B., Nitsch, R.M., McGaugh, J.L., and Hock, C. 2000. Acute cortisone administration impairs retrieval of long-term declarative memory in humans. Nat. Neurosci. 3: 313314.

Gold, P.E. and McGaugh, J.L. 1975. A single-trace, two process view of memory storage processes. In Short-term memory (eds. J. Deutsch and D. Deutsch), pp. 355-378. Academic Press, NY.

Lang, P.J., Bradley, M.M., and Cuthbert, B.N. 1999. International affective picture system (IAPS): Technical manual and affective ratings. The Center for Research in Psychophysiology, University of Florida, Gainesville, FL.

Livingston, R.B. 1967. Reinforcement. In The neurosciences: A study program (eds. G. Quarton, T. Melnechuk, and F. Schmitt), pp. 514-76. Rockefeller Press, NY.

Lovallo, W. 1975. The cold pressor test and autonomic function: A review and integration. Psychophysiology 12: 268-282.

Manning, C.A., Parsons, M.W., and Gold, P.E. 1992. Anterograde and retrograde enhancement of $24 \mathrm{~h}$ memory by glucose in elderly humans. Behav. Neural. Biol. 58: 125-130.

McGaugh, J.L. 2000. Memory-A century of consolidation. Science 287: 248-251.

Messier, C., Pierre, J., Desrochers, A., and Gravel, M. 1998. Dose-dependent action of glucose on memory processes in women: Effect on serial position and recall priority. Cogn. Brain Res. 7: 221-233.

Nielson, K.A., Radtke, R.C., and Jensen, R.A. 1996. Arousal-induced modulation of memory storage processes in humans. Neurobiol. Learn. Mem. 66: 133-142.

Roozendaal, B. 2000. Glucocorticoids and the regulation of memory consolidation. Psychoneuroendocrinol. 25: 213-238.

Scholey, A.B., Moss, M.C., and Wesnes, K. 1998. Oxygen and cognitive performance: The temporal relationship between hyperoxia and enhanced memory. Psychopharmacology (Berl) 140: 123-126.

Shors, T.J. 1998. Stress and sex effects on associative learning: For better or for worse. Neuroscientist 4: 353-364.

Soetens, E., Casaer, S., D'Hooge, R., and Hueting, J.E. 1995. Effect of amphetamine on long-term retention of verbal material. Psychopharmacology (Berl) 119: 155-162.

Southwick, S.M., Davis, M., Horner, B., Cahill, L., Morgan III, C.A., Gold, P.E., Bremner, J.D., and Charney, D.C. 2002. Relationship of enhanced norepinephrine activity during memory consolidation to enhanced long-term memory in humans. Am. J. Psychiatry 159: 1420-1422.

Weingartner, H. and Parker, E.S. 1984. Memory consolidation: Psychobiology of cognition. Lawrence Erlbaum Associates, Hillsdale, NJ.

Wolf, O.T., Shommer, N.C., Hellhammer, D.H., McEwen, B.S., and Kirschbaum, C. 2001. The relationship between stress induced cortisol levels and memory differs between men and women. Psychoneuroendocrinol. 26: 711-720.

Received April 17, 2003; accepted in revised form June 6, 2003. 


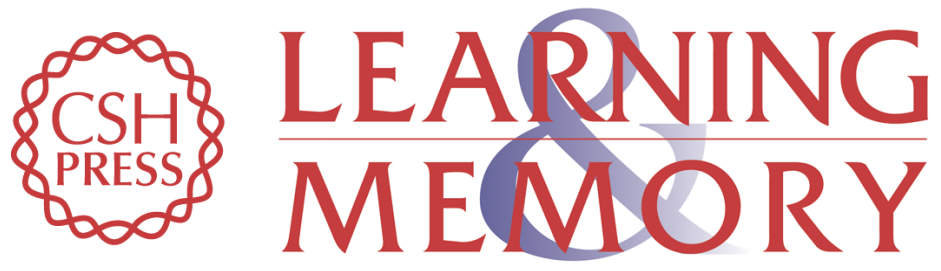

\section{Enhanced Human Memory Consolidation With Post-Learning Stress: Interaction With the Degree of Arousal at Encoding}

Larry Cahill, Lukasz Gorski and Kathryn Le

Learn. Mem. 2003, 10:

Access the most recent version at doi:10.1101/lm.62403

References This article cites 19 articles, 2 of which can be accessed free at:

http://learnmem.cshlp.org/content/10/4/270.full.html\#ref-list-1

License

Email Alerting Receive free email alerts when new articles cite this article - sign up in the box at the Service top right corner of the article or click here. 